which the "increased hydrophobicity" of picornaviruses brought about by certain treatments enables them to penetrate the "cell membrane" (P. Boulanger and K. Lonberg-Holm on Components of non-enveloped viruses which recognize receptors), which is reiterated by $\mathrm{R} . \mathrm{L}$. Crowell, B. J. Landau and J.-S. Siak (Picornavirus receptors in pathogenesis) in their reference to the fact that the "observation that 'A-particles' are more lipophilic than virions opens up the possibility that these particles fuse with the plasma membrane." Unfortunately, we are not likely to get a book on virus penetration in this series; could the present editors and the publishers, nevertheless, be persuaded to bring out such a book in the same format as Virus receptors?

In reading this book, one becomes struck by the apparent paradox that, while it is viral receptors that predominantly underly the cell specificity of viral infections, a very large number of receptors all appear to be of similar structure, i.e., sialic acid-containing glycoproteins. A. T. H. Burness (Glycophorin and sialylated components as receptors for viruses) attempts to resolve this point by suggesting that "sialic acid [may be] relegated to an indirect role in determining the critical structure of the sialo-glycoprotein", but one cannot help wondering whether all the in-vitro experiments on which the dominant role of sialic acid as viral receptor is based do indeed accurately reflect the situation in vivo. Equally surprising are the published association constants for binding between virus and receptor (N. L. Incardona: The chemical nature of virus-receptor interactions); is it really likely that these are some 10-1000 times stronger than antigen-antibody interactions? Is it not more likely that some of the underlying assumptions (e.g., that the system is at equilibrium and that $\mathrm{k}_{3}$ - the rate constant for the step subsequent to binding that commits a biral particle to entry-can be ignored) are at fault?

These comments illustrate the breadth of coverage in this book. In addition to the chapters mentioned, there are articles on subviral components of myxo- and paramyxo-viruses which recognise receptors (A. S. Scheid) and the biology and biochemistry of cellular receptors for enveloped viruses (K. V. Holmes), and on membrane components interacting with non-enveloped viruses (P. Boulanger and L. Philipson) and retrovirus receptors and their genetics (R. A. Weiss), two chapters that well illustrate how much less we know, in molecular terms, of the receptors on cells than of the "ligands" on the virus, in these two instances.

C. A. Pasternak

\title{
Interferon and interferon inducers. Clinical applications
}

Edited by D. A. Stringfellow. 1980. Marcel Dekker, Inc., New York. Pp. ix and 329. Sw.F. 92.

This is the 17th volume in the series Modern pharmacology-toxicology. It differs considerably from previous books dealing with interferons in that the editor has particularly emphasised the topics that he feels are most relevant to the use of interferons in medicine. These are considered in 10 chapters, written by specialists and uneven in their quality and coverage. The many other aspects of interferon research are covered briefly in an introductory and rather dated chapter (the latest papers reviewed are from 1978).

There has been much recent interest in the use of interferons, made from human cells or by recombinant DNA techniques, as antiviral or antitumour agents in man. It is therefore appropriate to be reminded in the book of an attractive alternative, namely to use chemical agents which, when injected, stimulate the body to form its own interferon. Dr Stringfellow provides an excellent general chapter on such interferon inducers, which reflects his considerable interest and experience in this area, and this is one of the best things in this book. Four chapters then deal in great detail with particular classes of inducers. Although these will provide a reference source for the specialist, they are over-detailed for the general reader. For example, a 36-page account of Tilorone, which the authors (and discoverers of this agent) dismiss in their last sentence as a laboratory curiousity, seems excessive. It is important to remember that, while interferons are physiological substances, chemicals that induce their formation are not, and toxicities and side effects may be encountered. The polyanions reviewed by Dr Breinig and Dr 
Morahan, have a wide range of pharmacological effects and toxicities, and as Dr Levy points out, the inducer, poly-ICLC, is an immune stimulant, whereas interferons are in general immunosuppressive.

Of the other chapters, that by Dr Billiau and Dr DeSomer provides an outstanding critique of what has been achieved by the use of interferon as an antiviral agent in animals and man, and what might be achieved. This should be required reading for any clinician with access to interferon. In contrast, the chapter on the antitumour uses of interferon is a disappointment. Other chapters deal with the production, purification and properties of human interferons, the stability and pharmacokinetics of interferons, and effects of interferon and inducers on the immune system. This latter is a useful account of what remains a very complicated field, even though the recent work on interferon as a stimulator of natural killer cells is only briefly mentioned.

As a reference source for the experienced interferon researcher (one of the avowed aims of the editor) this book will be useful. The other aim, to provide a work broad enough to be of value to the novice (surely incompatible with the first aim), has not been met. Moreover, in view of the price, this is a book that few will want to own, which is a pity because some excellent chapters will not be as widely read as they deserve. This is not a book for the non-specialist to buy, even though, like the curate's egg, parts are excellent.

N. B. FINTER

\section{Hepatitis B virus and primary hepatocellular carcinoma}

Edited by Ph Maupas and J L. Melnick. 1981. Progress in medical virology, vol. 27. S. Karger, Basle. Pp. viii and 210. US\$75.00.

Primary hepatocellular carcinoma is a very common and important tumour, particularly among young males, in many regions of Africa, South-East Asia, China and Japan. The annual incidence is estimated as 350000 cases. There is now compelling epidemiological and virological evidence of an aetiological association between hepatitis B virus and this tumour. Some of the evidence was thoroughly reviewed during a workshop that was organised by Professor Ph Maupas in April 1980 in Dakar. A few months later Philippe Maupas tragically lost his life in a motorcar accident.

The topics covered in this volume of the proceedings include studies on the epidemiology of primary hepatocellular carcinoma and hepatitis B infection in Senegal, South Africa, Taiwan and Greece, the localisation of hepatitis B viral antigens in liver tissue, integration of hepatitis B viral DNA sequences in the tumour tissue and studies on cell lines derived from hepatocellular carcinoma. The preparation of the 22-nm spherical particle hepatitis B vaccine in France is described, as well as the design of a vaccine field trial in Senegal and implementation of the programme among pregnant women and children under 2 years old for the prevention of perinatal infection and infection during infancy. There are also two useful papers on comparative epidemiology and pathogenesis of cancers associated with viruses in man and animals.

Although the exact mechanism of malignant transformation of hepatocytes in not yet known, infection with hepatitis B virus appears to trigger the chain of cellular changes. It should be noted, however, that other factors may play an important role in the aetiology of primary hepatocellular carcinoma, including genetic background, hormones, the immune response, nutrition and environmental carcinogens such as alfatoxin and other mycotoxins, chemical carcinogens and alcohol and apparently even cigarette smoking in hepatitis B-negative hepatocellular carcinoma.

Many readers, particularly those with less specialised knowledge, will regret that some of the interesting studies on the expression of hepatitis B virus by cloning the viral genome in eukaryotic and prokaryotic cells are described only in telegraphic form, although the details have been published elsewhere. A far less serious criticism is the extensive use by some authors of inconsistent or unconventional abbreviations. For example, PHC (primary hepatocellular 